OPEN ACCESS

Edited by:

Tiago Collares,

Federal University of Pelotas, Brazi

Reviewed by:

Carmelo Laudanna

Institute for Research in Biomedicine,

Spain

Haiwei Mou

Cold Spring Harbor Laboratory,

United States

${ }^{*}$ Correspondence:

Guang Li

L17609833084@163.com

Specialty section:

This article was submitted to

Cancer Genetics,

a section of the journal

Frontiers in Genetics

Received: 22 November 2019

Accepted: 26 March 2020

Published: 29 April 2020

Citation:

Long H, LiU J, Yu Y, Qiao Q and Li G (2020) PKMYT1 as a Potential Target to Improve the Radiosensitivity

of Lung Adenocarcinoma.

Front. Genet. 11:376.

doi: 10.3389/fgene.2020.00376

\section{PKMYT1 as a Potential Target to Improve the Radiosensitivity of Lung Adenocarcinoma}

\author{
Huan-ping Long, Jia-qing Liu, Yang-yang Yu, Qiao Qiao and Guang Li* \\ Department of Radiation Oncology, The First Affiliated Hospital of China Medical University, Shenyang, China
}

Objective: This article is dedicated to finding important genes related to the prognosis of lung adenocarcinoma (LUAD), looking for a new gene that may affect tumor radiosensitivity, and conducting basic experiments to verify the relationship between this gene and the radiosensitivity of LUAD.

Methods: The gene expression profiles GSE32863, GSE33532, and GSE43458 were obtained from NCBI-GEO. GEO2R and a Venn diagram were used to identify upregulated genes. STRING and Cytoscape were applied to develop a protein-protein interaction network (PPI) and analyze the modules. The Database for Annotation, Visualization and Integrated Discovery (DAVID) was used to process the GO and KEGG pathway analysis. The Kaplan Meier plotter and Gene Expression Profiling Interactive Analysis (GEPIA) were applied to get the significant prognostic information and differential expression between LUAD tissues and normal lung tissues. Western blotting and Q-PCR were used to detect the expression of PKMYT1 in tissues. Small interfering RNAs (siRNAs) were used to knockdown PKMYT1. The colony survival experiment was used to assess the effect of PMYT1 on the radiosensitivity of tumor cells. Cell cycle analysis was used to assess cell cycle distribution.

Results: We identified 14 genes (PKMYT1, TTK, CHEK1, CDC20, PTTG1, MCM2, CDC25C, MCM4, CCNB1, CDC45, MAD2L1, CCNB2, BUB1, and CCNA2) that are important for LUAD and may be potential therapeutic targets. We confirmed that PKMYT1 is highly expressed in LUAD and firstly demonstrated that artificially silencing the expression of PKMYT1 can abrogate IR-induced G2/M phase arrest and increase the sensitivity of cancer cells to radiation.

Conclusion: In summary, we obtained 14 core genes related to the poor prognosis of LUAD via bioinformatical analysis. We identified that PKMYT1 was significantly upregulated in LUAD tissues and firstly demonstrated that knockdown of PKMYT1 can eliminate the radiation-induced G2/M arrest, resulting in a lower survival rate for cells receiving radiation therapy. Our findings suggested that PKMYT1 is a promising target to improve the radiosensitivity of LUAD.

Keywords: bioinformatics, PKMYT1, G2 checkpoint, lung adenocarcinoma, radioresistance 


\section{INTRODUCTION}

Lung cancer accounts for $18.4 \%$ of cancer deaths and remains the leading cause of cancer-related deaths (Bray et al., 2018). Lung adenocarcinoma has become one of the most common types of lung cancer in recent decades (Siegel et al., 2018). Radiotherapy is an effective treatment of LUAD, especially when some patients are not suitable for surgery. However, LUAD from different patients may display different degrees of radiation tolerance. Radioresistance is the main factor reducing the effectiveness of

Abbreviations: DAVID, the Database for Annotation, Visualization and Integrated Discovery; GO, Gene Ontology; GEPIA, the Gene Expression Profiling Interactive Analysis; KEGG, Kyoto Encyclopedia of Genes and Genomes; LUAD, lung adenocarcinoma; PPI, protein-protein interaction; OS, overall survival. radiotherapy, resulting treatment failure (Provencio et al., 2010; Le Pechoux, 2011). Thus, it is important to conduct researches on developing radiosensitizers to treat this lethal disease.

Bioinformatics can help us explore a lot of valuable clues, find meaningful genes, and conduct new research. In the present study, GSE32863, GSE33532, and GSE43458 were analyzed through bioinformatics. Finally, we obtained fou 14 genes (PKMYT1, TTK, CHEK1, CDC20, PTTG1, MCM2, CDC25C, MCM4, CCNB1, CDC45, MAD2L1, CCNB2, BUB1, and CCNA2), which were significantly enriched in the cell cycle.

After an extensive literature review of these 14 genes, we found that PKMYT1 is a highly promising gene that may be closely related to tumor radiosensitivity. This is because studies have found that the abrogation of G2 checkpoint effectively

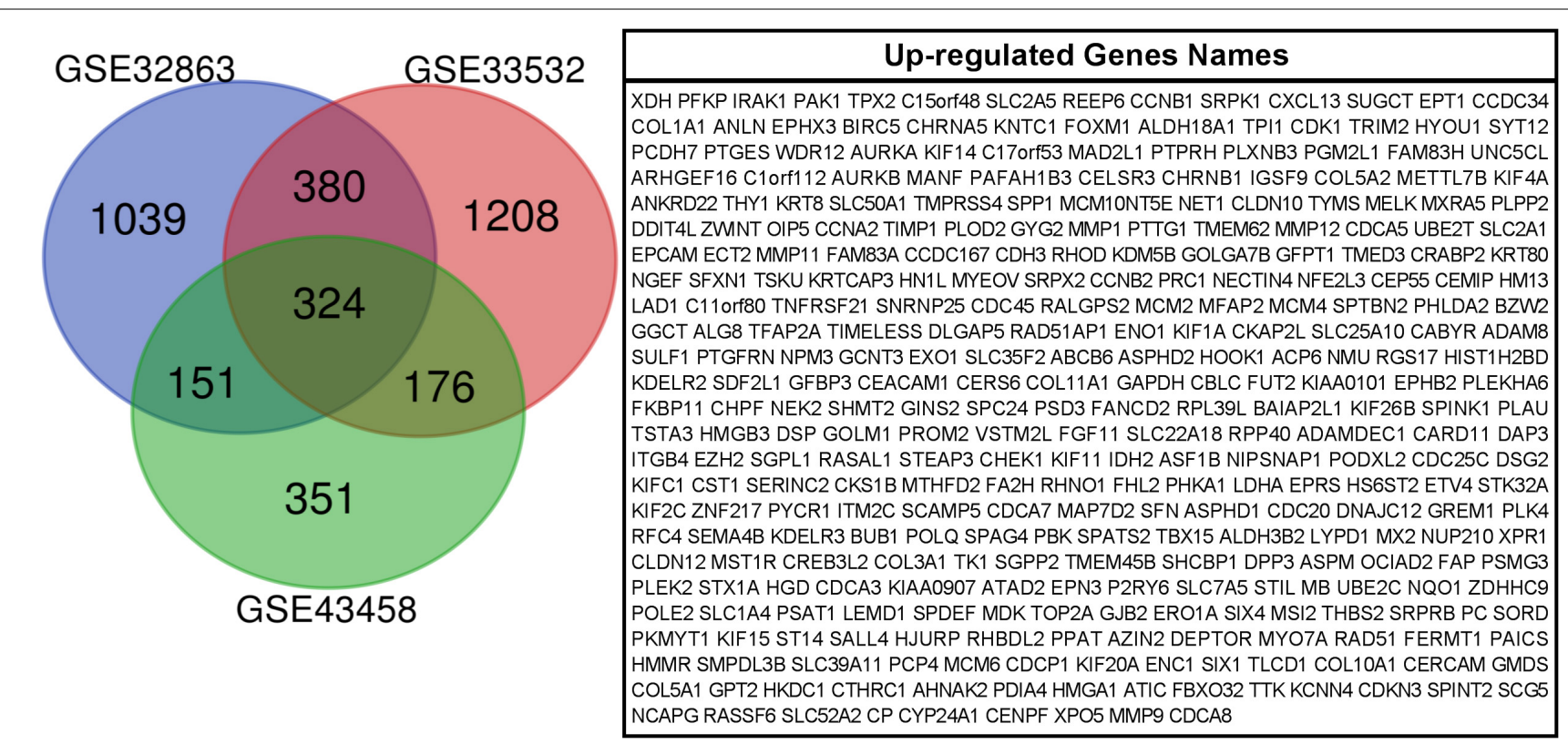

FIGURE 1 | 324 commonly up-regulated genes were obtained from GSE33532, GSE43458and GSE 32863 via GEO2R online tools and Venn diagram software.

TABLE 1 | Gene ontology analysis of 324 genes.

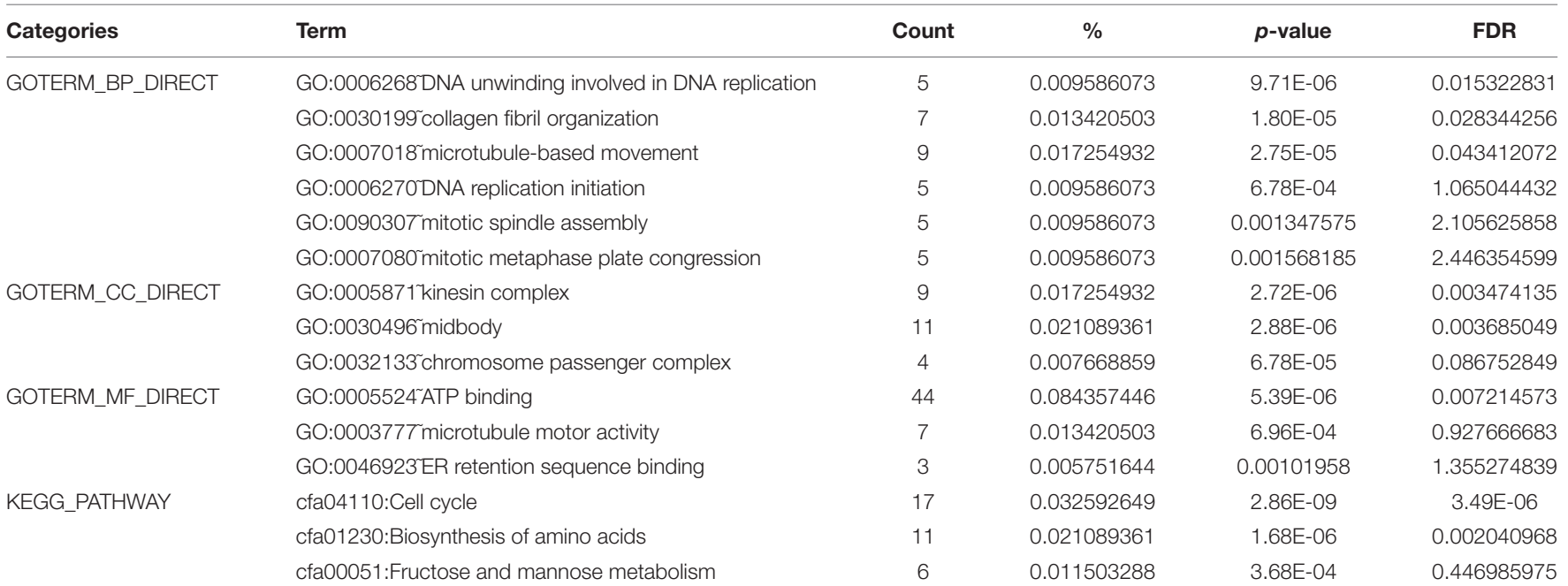




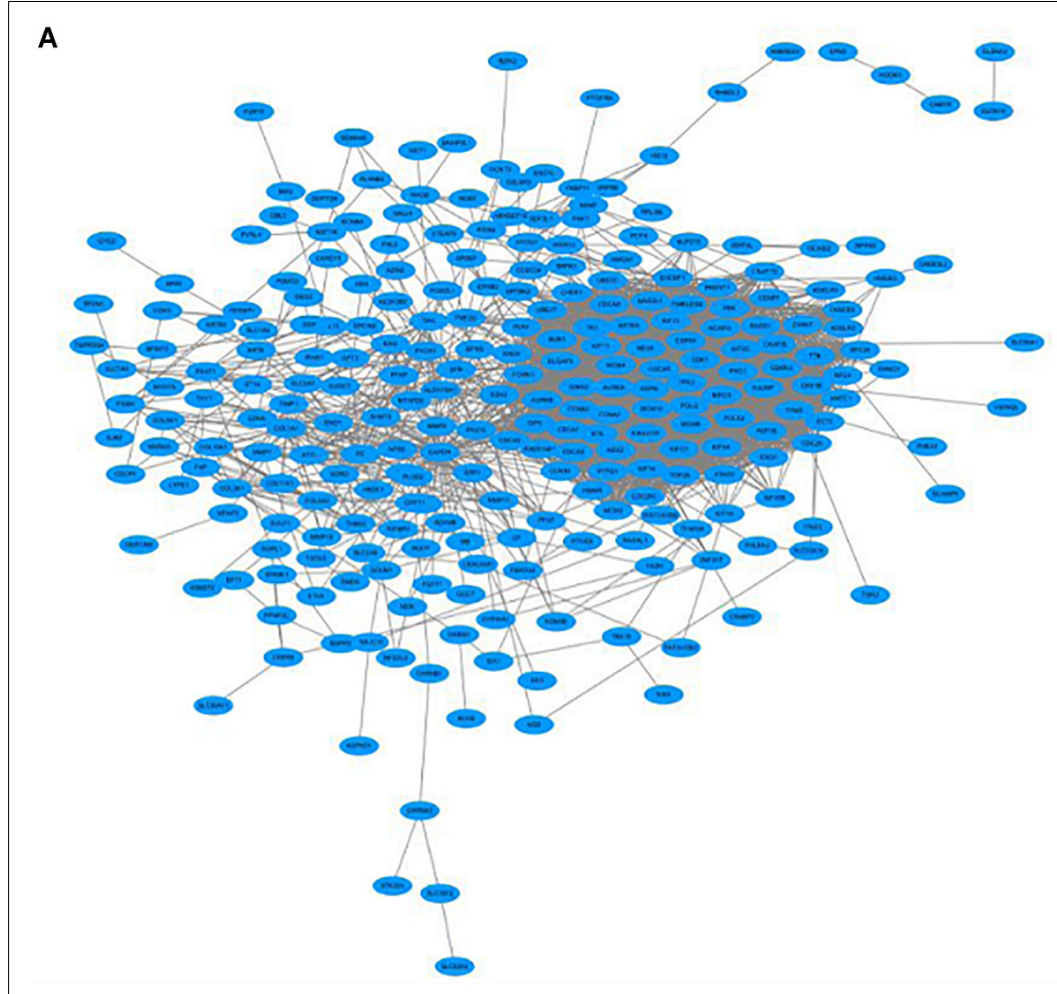

B

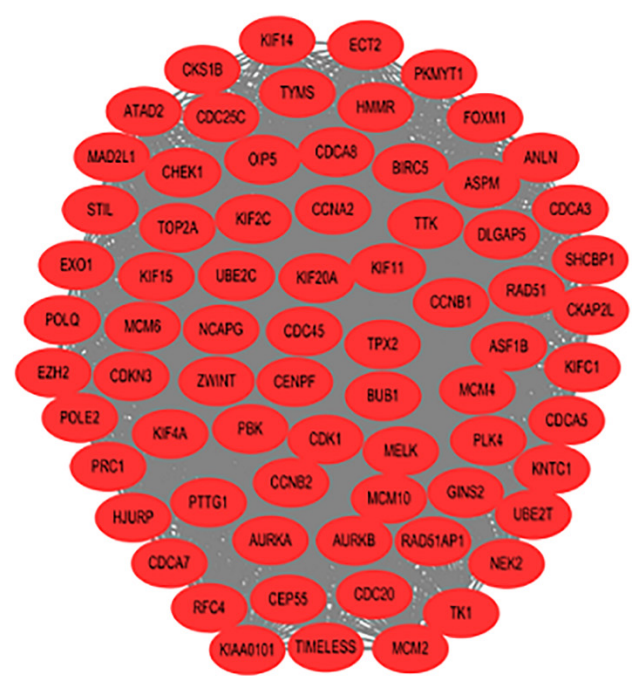

FIGURE 2 | (A) Construction of the protein-protein interaction (PPI) network. (B) Identification of the significant module.

reduced radiation-induced cell cycle arrest and increased tumor radiosensitivity (Leijen et al., 2010; De Witt et al., 2011; Qin et al., 2014; Busch et al., 2017). Additionally, PKMYT1 is currently considered to be the target of G2 checkpoint elimination and mitotic catastrophe (Tse et al., 2009; Schmidt et al., 2017) because PKMYT1 can prevent cells from transitioning from G2 to mitosis phase in two ways. One is inhibiting Cdk1 activity via phosphorylating Cdk1 at Thr14 and Tyr15, and the other is preventing the Cdk1-CycB complex from entering the nucleus by binding to the Cdk1-CycB complex and sequestering it in the cytoplasm (Fattaey and Booher, 1997; Wells et al., 1999; Passer et al., 2003).

TABLE 2 | The overall survival analysis of the 72 hub genes.

\begin{tabular}{ll}
\hline Categories & Genes names \\
\hline Genes with significantly & MAD2L1 PKMYT1 KIF2C TTK CCNA2 BUB1 \\
poor OS $(P<0.05)$ & KIF20A CKAP2L BIRC5 CCNB2 EXO1 CDCA3 \\
& CDKN3 CDC20 OIP5 HJURP KIF14 MCM2 \\
& MCM10 PRC1 MELK KIF15 NCAPG PTTG1 TK1 \\
& KIF4A UBE2C FOXM1 AURKA KIFC1 CEP55 \\
& CDC25C TYMS ASF1B KIAA0101 EZH2 CENPF \\
& DLGAP5 CHEK1 CDCA5 ZWINT ANLN POLQ \\
& CDC45 GINS2 TIMELESS SHCBP1 RFC4 NEK2 \\
& AURKB UBE2T CCNB1 TPX2 MCM4 TOP2A PBK \\
& ASPM CDCA8 \\
& KNTC1 HMMR RAD51 POLE2 ECT2 RAD51AP1 \\
& STIL ATAD2 PLK4 CDK1 KIF11 MCM6 CDCA7 \\
Genes without & CKS1B \\
significantly poor OS &
\end{tabular}

However, there is no experimental evidence to prove the relationship between PKMYT1 and tumor radiosensitivity. We designed experiments to see if PKMYT1 can affect the resistance of LUAD cells to radiation, providing important evidence for PKMYT1 as a radiosensitizer in radiotherapy.

\section{MATERIALS AND METHODS}

\section{Bioinformatics Analysis}

The GSE32863, GSE33532, and GSE43458 were obtained from NCBI-GEO, which included 40 LUAD tissues and 10 normal lung tissues, 80 LUAD tissues and 30 normal lung tissues, and 58 LUAD tissues and 58 normal lung tissues, respectively. GEO2R was used to identify upregulated genes with $\operatorname{logFC}>0.5$ and an adjusted $P<0.001$. Venn diagram was used to select upregulated genes co-expressed in these three gene chips. A PPI network was established by STRING. Then, we use the Cytoscape to find potential relevance between those genes. Next, we used MCODE (Molecular Complex Detection) to obtain core genes. We processed BP, MF, CC, and KEGG pathway analyses $(P<0.05)$ using the Database for Annotation, Visualization and Integrated Discovery (DAVID). The Kaplan Meier plotter and Gene Expression Profiling Interactive Analysis (GEPIA) were applied to get the significant prognostic information $(P<0.05)$ of hub genes in LUAD and their differential expression between LUAD tissues and normal lung tissues $(P<0.05)$. 


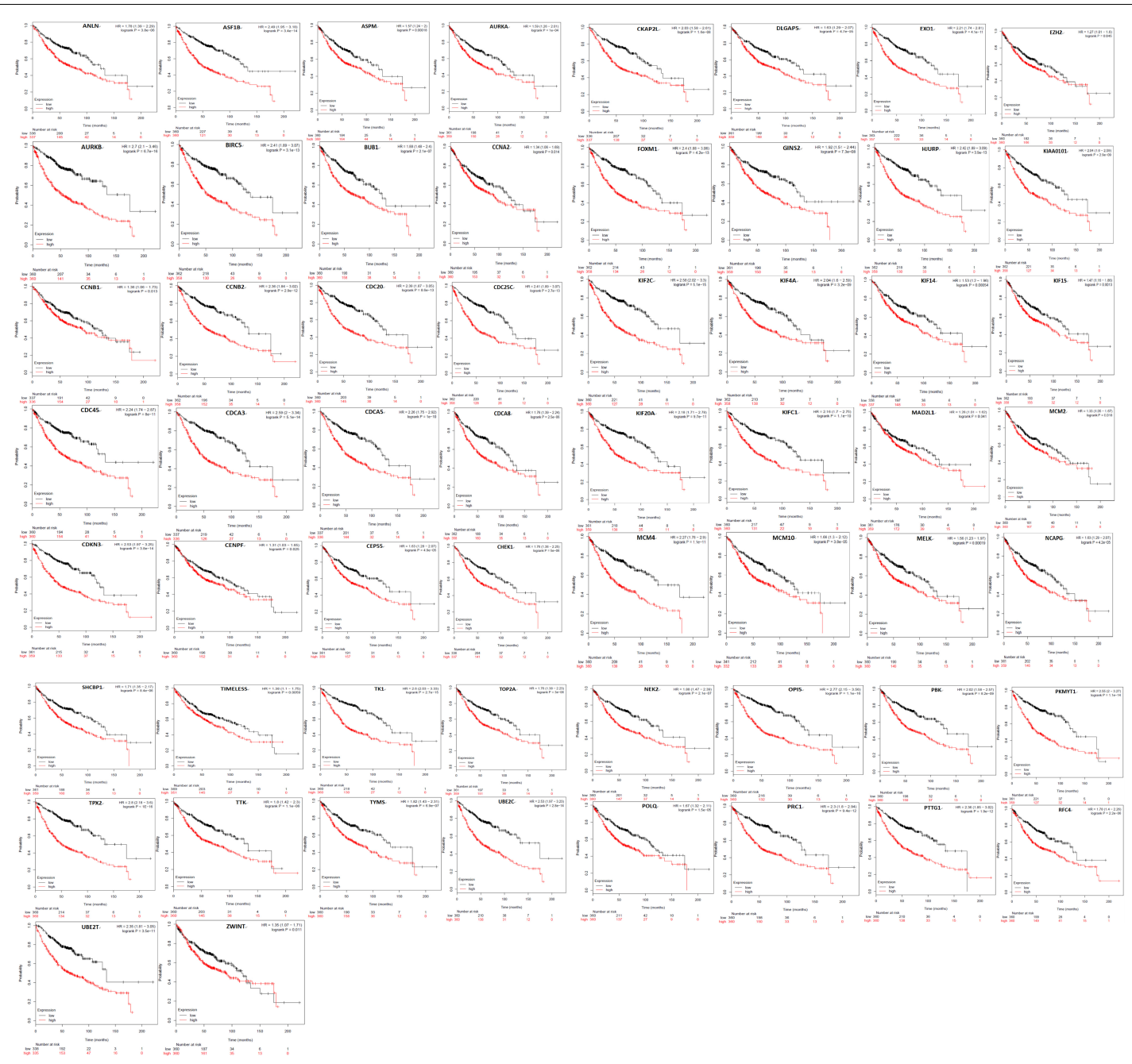

FIGURE 3 | A total of 58 of 72 genes had a significantly poor survival rate in LUAD via Kaplan meier plotter online tools $(P<0.05)$.

\section{Human LUAD Specimens}

Human LUAD tissues with the matched normal adjacent specimens were obtained from The First Hospital of China Medical University. This study was approved by the Ethics Committee of The First Hospital of China Medical University.

\section{Cell Culture}

LUAD cell lines: A549, H299, H1975, H1650, and H441 were cultured using Roswell Park Memorial Institute-1640 (RPMI1640 , Hyclone) medium containing $10 \%$ fetal bovine serum (FBS, Clarks) at $37^{\circ} \mathrm{C}$.

\section{siRNA and Transfection}

Small interfering RNAs (siRNAs) against PKMYT1 (siRNA\#1 and siRNA\#2) and negative control siRNAs (NC-siRNA), which were obtained from Shanghai GENECHEM Co., Ltd. (Jikai, Shanghai, China), were transfected into A549 and H1299 cells using Neofect reagent (ProbeGenne, Jiangsu, China) according to the manufacturer's instructions.

\section{Quantitative PCR (Q-PCR)}

Reverse transcription was carried out according to the instructions of PrimeScript ${ }^{\mathrm{TM}} \mathrm{RT}$ reagent Kit with GNA

TABLE 3 | Differential expression of 53 genes between LUAD and normal lung tissues.

Categories

\section{Genes names}

Genes with significantly high expression in LUAD $(P<0.05)$ significantly high expression in LUAD $(P<0.05)$
MAD2L1 PKMYT1 KIF2C TTK CCNA2 BUB1 KIF2OA CKAP2L BIRC5 CCNB2 EXO1 CDCA3 CDKN3 CDC20 OIP5 HJURP MCM2 MCM10 PRC1 MELK KIF15 NCAPG PTTG1 TK1 KIF4A UBE2C FOXM1 AURKA KIFC1 CEP55 CDC25C TYMS ASF1B KIAA0101 CENPF DLGAP5 CHEK1 CDCA5 ZWINT ANLN CDC45 GINS2 SHCBP1 NEK2 AURKB UBE2T CCNB1 TPX2 MCM4 TOP2A PBK ASPM CDCA8

KIF14 EZH2 POLQ TIMELESS RFC4 


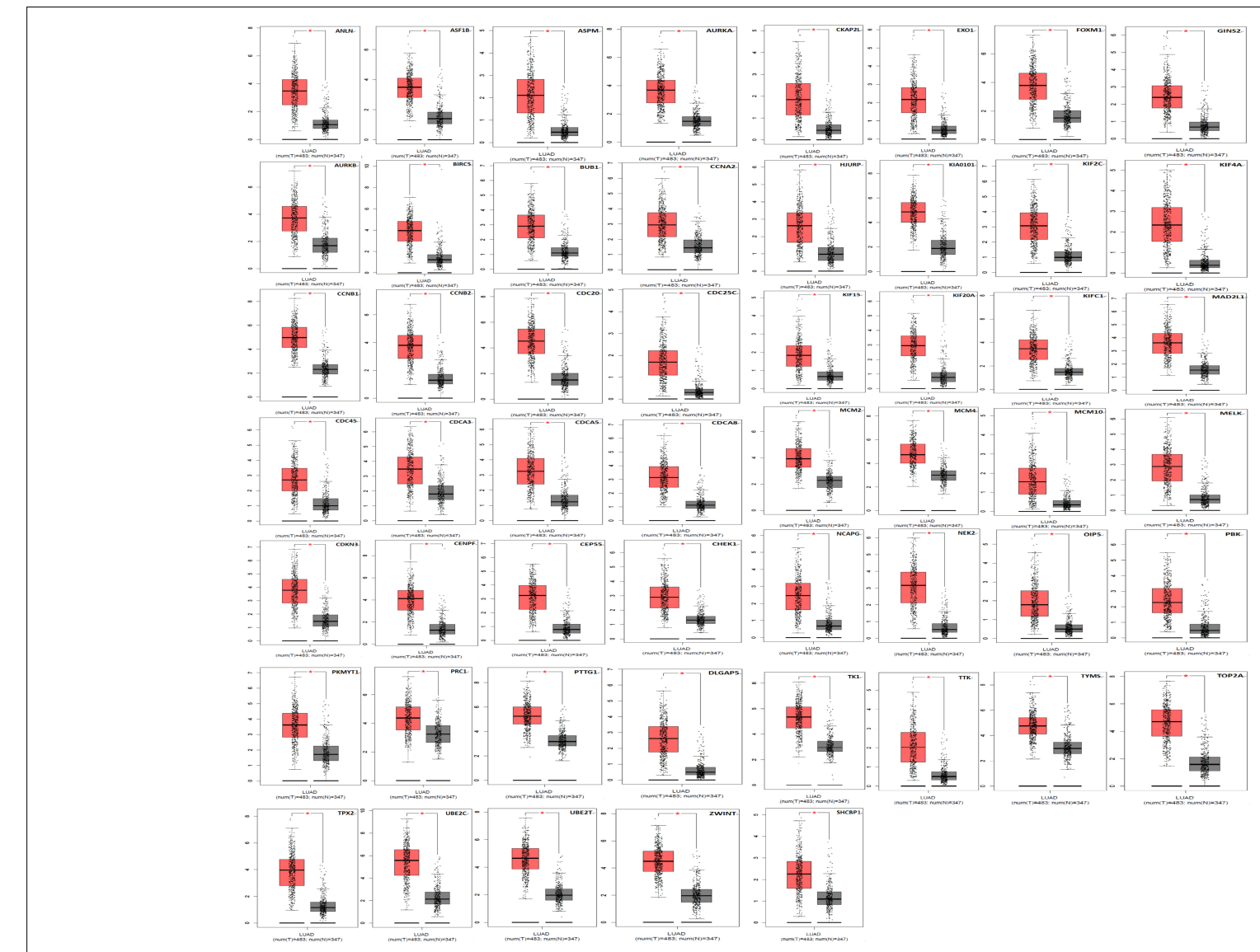

FIGURE 4 | A total of 53 of 58 genes are significantly upregulated in LUAD samples contrasted to normal lung samples $\left({ }^{*} P<0.05\right)$.

Eraser (Takara), and SYBR Premix Ex TaqII (Takara) was used for Q-PCR. We used several sequences: PKMYT1 forward primer $5^{\prime}$-CATGGCTCCTACGGAGAGGT-3' and reverse primer $5^{\prime}$-ACATGGAACGCTTTACCGCAT- $3^{\prime} ; \beta$-actin forward primer $5^{\prime}$-CCAGACAGCACTGTGTTGGCATA-3', and reverse primer 5'-ATGTTGCCCTAGACTTCGAGCAAG-3. The results were calculated using the $2^{-\Delta \Delta \mathrm{Ct}}$ method.

\section{Western Blotting Assay}

The protein samples were loaded onto SDS-PAGE, transferred onto PVDF membranes, and immunoblotted with primary antibodies against PKMYT1 (Abcam) and $\beta$-actin (CST) and peroxidase-labeled secondary antibodies (CST). The Enhanced Chemiluminescence System (OLYMPUS) was used to display immune response bands.

\section{Irradiation Treatment and Clonogenic Survival Assay}

Transfected A549 and H1299 cells were irradiated with 0, 2, 4, 6 , and 8 Gy X-rays. After 12 days, the cells were fixed with $4 \%$ paraformaldehyde and stained with $0.1 \%$ crystal violet solution. A single- hit multi-target model formula: $S F=1-\left(1-e^{-D / D 0}\right)^{n}$ was used to fit cell survival curves and calculate parameters (D0, $\mathrm{Dq}, \mathrm{k}, \mathrm{N}$, and SER).
TABLE 4 | KEGG pathway enrichment analysis of 53 genes.

\begin{tabular}{|c|c|c|c|c|}
\hline Term & Count & $\%$ & $p$-value & genes \\
\hline $\begin{array}{l}\text { cfa04110:Cell } \\
\text { cycle }\end{array}$ & 14 & 0.15171218 & $2.29 \mathrm{E}-18$ & $\begin{array}{l}\text { PKMYT1, TTK, } \\
\text { CHEK1, CDC20, } \\
\text { PTTG1, MCM2, } \\
\text { CDC25C, MCM4, } \\
\text { CCNB1, CDC45, } \\
\text { MAD2L1, } \\
\text { CCNB2, BUB1, } \\
\text { CCNA2 }\end{array}$ \\
\hline $\begin{array}{l}\text { cfa04114:Oocyte } \\
\text { meiosis }\end{array}$ & 8 & 0.086692674 & $2.13 \mathrm{E}-08$ & $\begin{array}{l}\text { CCNB2, } \\
\text { MAD2L1, BUB1, } \\
\text { PKMYT1, } \\
\text { CDC20, AURKA, } \\
\text { PTTG1, CDC25C }\end{array}$ \\
\hline $\begin{array}{l}\text { cfa04914: } \\
\text { Progesterone- } \\
\text { mediated } \\
\text { oocyte } \\
\text { maturation }\end{array}$ & 7 & 0.07585609 & 1.67E-07 & $\begin{array}{l}\text { CCNB1, CCNB2, } \\
\text { MAD2L1, BUB1, } \\
\text { PKMYT1, } \\
\text { CDC25C, CCNA2 }\end{array}$ \\
\hline
\end{tabular}

\section{Cell Cycle Analysis}

Cells transfected with siRNA \# 1, siRNA \# 2, and NCsiRNA were irradiated with X-rays at different doses (0, 4Gy). After irradiation, the cells were further cultured for $24 \mathrm{~h}$. 
A

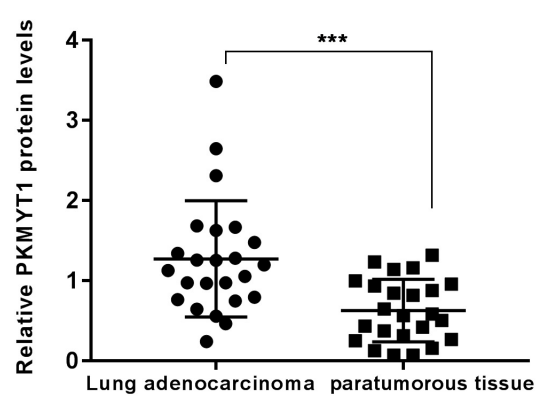

B

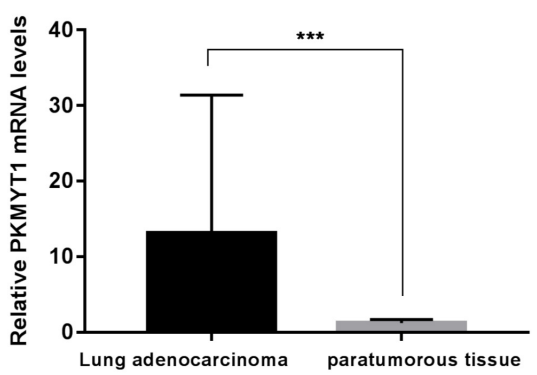

C
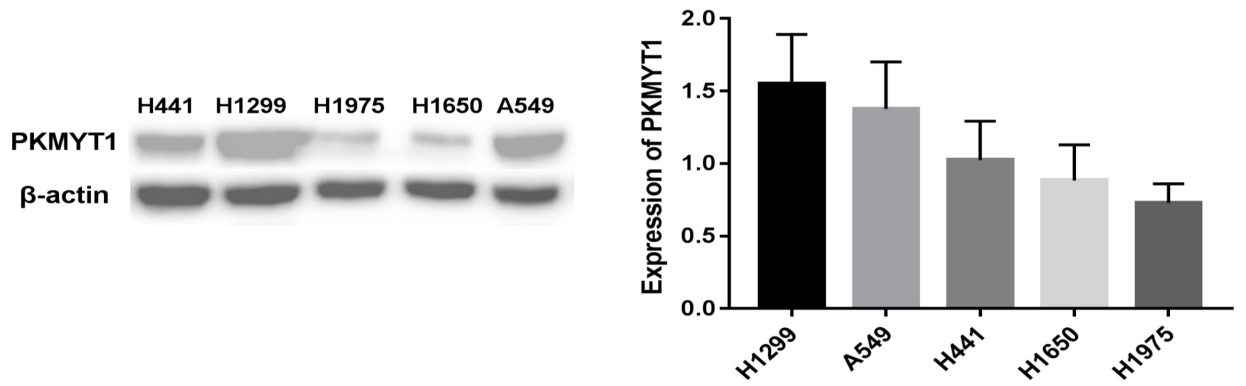

FIGURE 5 | PKMYT1 is highly expressed in lung adenocarcinoma tissues. (A) Relative PKMYT1 protein levels in 24 pairs of lung adenocarcinoma tissues and their matched normal tissues were determined by western blotting. (B) Relative PKMYT1 mRNA levels in 33 pairs of lung adenocarcinoma tissues and their matched normal tissues were determined by Q-PCR. (C) Expression level of PKMYT1 in five lung adenocarcinoma cell lines. ${ }^{\star \star *} p<0.001$.

The cells were trypsinized, washed with PBS, fixed with icecold $70 \%$ ethanol, and stored at $4^{\circ} \mathrm{C}$ for $12-24 \mathrm{~h}$. Prior to analysis by flow cytometry, the cells were washed with PBS, treated with $0.25 \mathrm{mg} / \mathrm{ml}$ RNase $\mathrm{A}$, and $50 \mu \mathrm{g} / \mathrm{ml}$ propidium iodide for $30 \mathrm{~min}$ at $37^{\circ} \mathrm{C}$. Next, the cells were subjected to cell cycle analysis using a fluorescence-activated cell sorter (BD FACS Calibur).

\section{Statistical Analysis}

Statistical analysis was conducted by SPSS 20.0 software. The statistical significance between groups was determined using a two-tailed Student's $t$-test. One-way ANOVA was used to compare different time points/dose within the same group. $P<0.05$ were considered to indicate statistically significant differences.

\section{RESULTS}

\section{Identification of Upregulated Genes in LUAD}

We used GEO2R to filter 2707, 1082, and 2085 upregulated genes from GSE33532, GSE43458, and GSE32863, respectively. We identified 324 commonly upregulated genes in the above datasets via Venn diagram software (Figure 1).

\section{Analyses of Gene Ontology and Pathway Enrichment}

DAVID was used to analyze the KEGG pathway and GO analysis of 324 genes. The GO analysis showed that, for biological processes (BP), genes significantly enriched DNA unwinding involved in DNA replication, collagen fibril organization, microtubule-based movement, DNA replication initiation, mitotic spindle assembly, and mitotic metaphase plate congression. For molecular function (MF), genes were primarily enriched in ATP binding, microtubule motor activity, and ER retention sequence binding. For cellular component (CC), genes were particularly enriched in the kinesin complex, midbody, and chromosome passenger complex. The results of the KEGG pathway analysis indicated that the 324 genes were significantly enriched in the cell cycle, biosynthesis of amino acids, and fructose and mannose metabolism (Table 1).

\section{PPI Network and Module Analysis}

A PPI network was constructed which included 267 nodes and 3161 edges. Then, a significant module with 72 nodes and 2357 edges was identified via MCODE (Figure 2).

\section{Identification of Core Genes}

Kaplan Meier plotter was used to analyze the overall survival (OS) of 72 hub genes. We identified that 58 genes had a significantly 
A

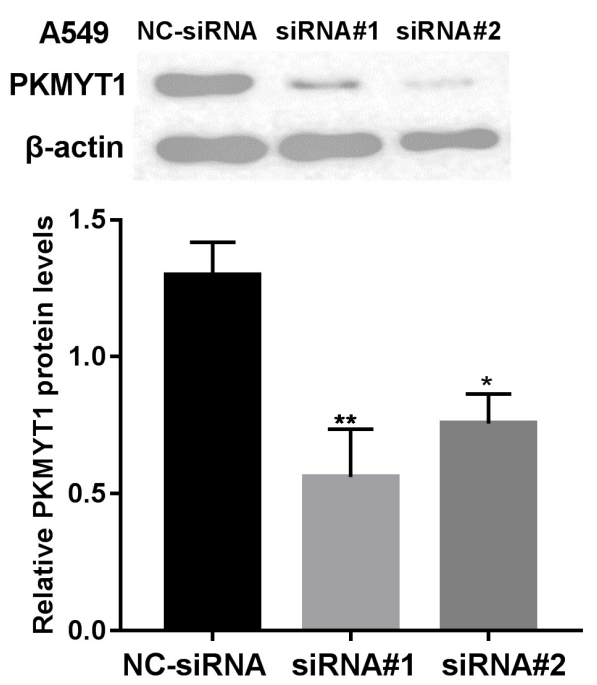

B

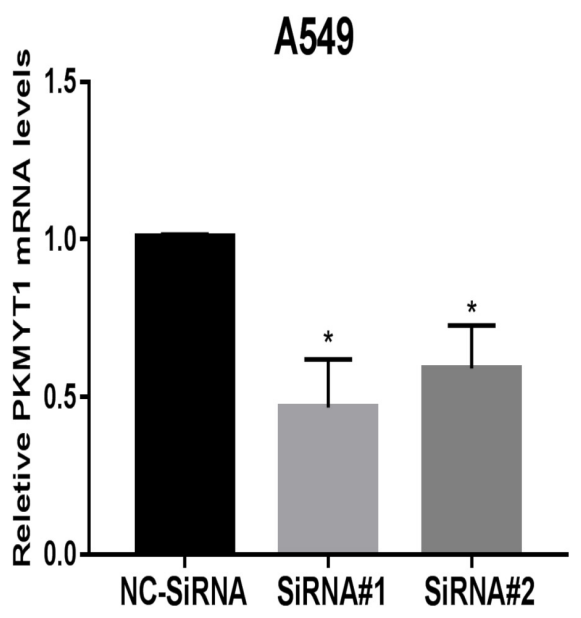

H1299 NC-siRNA SiRNA\#1 siRNA\#2

PKMYT1

$\beta$-actin
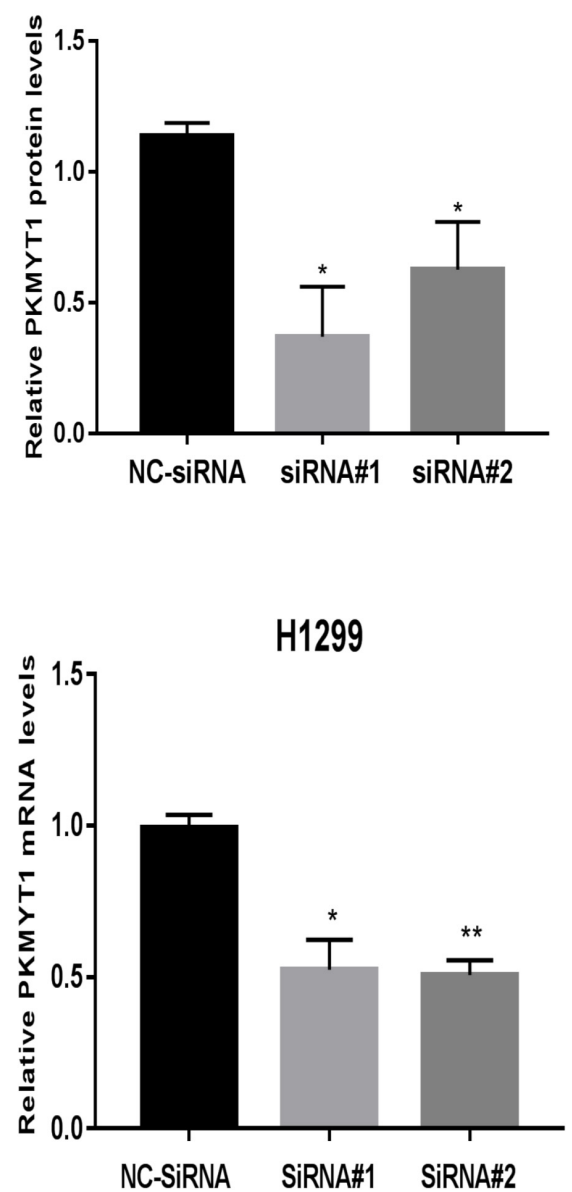

FIGURE 6 | Silencing efficiency of PKMYT1 in A549 and H1299 cells. (A) Western blotting assays detected the relative PKMYT1 protein levels in A549 and H1299 cells after transfecting with siRNA\#1, siRNA\#2, and NC-siRNA. (B) Q-PCR assays detected the relative PKMYT1 mRNA levels in A549 and H1299 cells after transfecting with siRNA\#1, siRNA\#2, and NC-siRNA. ${ }^{*} p<0.05,{ }^{\star *} p<0.01$.

poor OS in LUAD (Table 2 and Figure 3). Next, we found that 53 of 58 genes are highly expressed in LUAD tissues compared to normal lung tissues (Table 3 and Figure 4) via GEPIA.

\section{KEGG Pathway Analysis}

The results of the KEGG pathway analysis indicated that the 53 genes were significantly enriched in cell cycle, oocyte meiosis, and progesterone-mediated oocyte maturation. Results showed that fourteen genes (PKMYT1, TTK, CHEK1, CDC20, PTTG1, MCM2, CDC25C, MCM4, CCNB1, CDC45, MAD2L1, CCNB2, BUB1, and CCNA2) enriched in cell cycle (Table 4).

After an extensive literature review of these 14 genes, we found that PKMYT1 is a highly promising gene that may be closely related to tumor radiosensitivity. However, there is no experimental evidence to prove the relationship between PKMYT1 and tumor radiosensitivity. We therefore conducted basic experiments to explore whether PKMYT1 can affect tumor radiosensitivity.

\section{PKMYT1 Is Highly Expressed in Tumors Contrasted to Normal Tissues Derived From LUAD Patients}

Figure 5A reveals that the protein levels of PKMYT1 were distinctly increased in LUAD tissues compared to matched normal lung tissues via western blotting. Figure 5B shows that the mRNA levels of PKMYT1 in LUAD tissues were significantly higher than those in adjacent normal lung tissues by Q-PCR. 


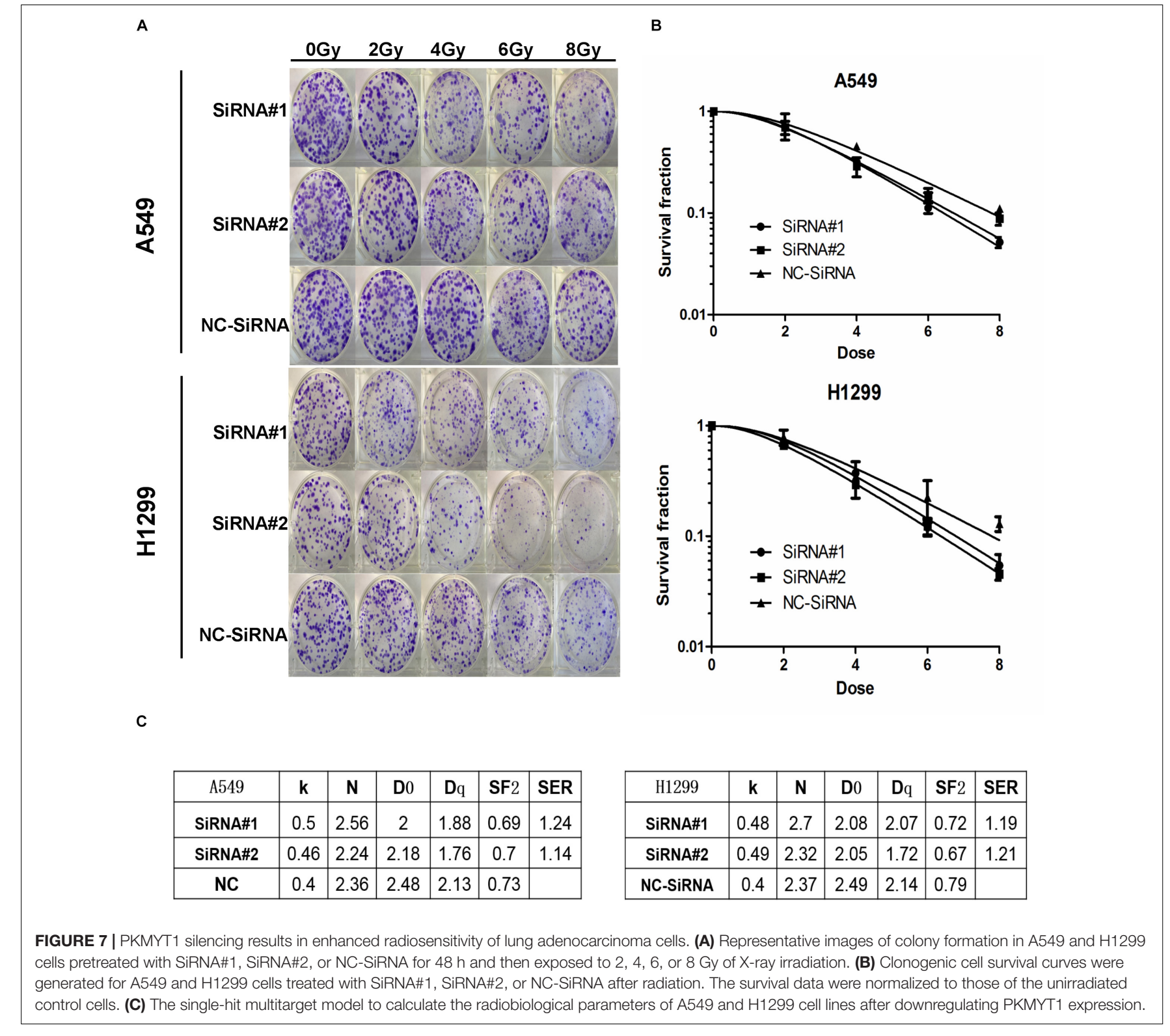

B

\section{siRNA Mediated the Silence of PKMYT1}

The expression of PKMYT1 in LUAD cell lines, including A549, H299, H1975, H1650, and H441, was confirmed by WB (Figure 5C). We next use the two most expressive ones (A549 and H1299 cells) for follow-up experiments. Both cells were infected with either control or PKMYT1 siRNA. Then, the Q-PCR and western blotting analyses were conducted, and the data suggested that siRNAs can significantly inhibit the expression of PKMYT1 in RNA and protein levels (Figures 6A,B).

\section{Downregulation of PKMYT1 Is Significantly Associated With Radiosensitivity of LUAD Cells}

The radiosensitivity of transfected A549 and H1299 cells was examined by colony formation assay. The data in Figure 7 demonstrates that artificial silencing of PKMYT1 remarkably enhanced radiosensitizing properties in LUAD Cells.

\section{Downregulation of PKMYT1 Alters Cell Cycle Distribution and Abrogates IR-Induced G2/M Phase Arrest}

As shown in Figures 8, 9, silencing of PKMYT1 can decrease the G2/M cell proportion in A549 and H1299, and the difference was statistically significant $(P<0.05)$. In both cell lines, following treatment with silencing of PKMYT1 + radiation (4Gy of X-rays), the cells also accumulated in G2/M, but to a much lower level compared to the radiation control $(p<0.05)$, suggesting that the silencing of PKMYT1 can eliminate the radiation-induced G2/M arrest, thereby improving the radiosensitivity of tumor cells. 


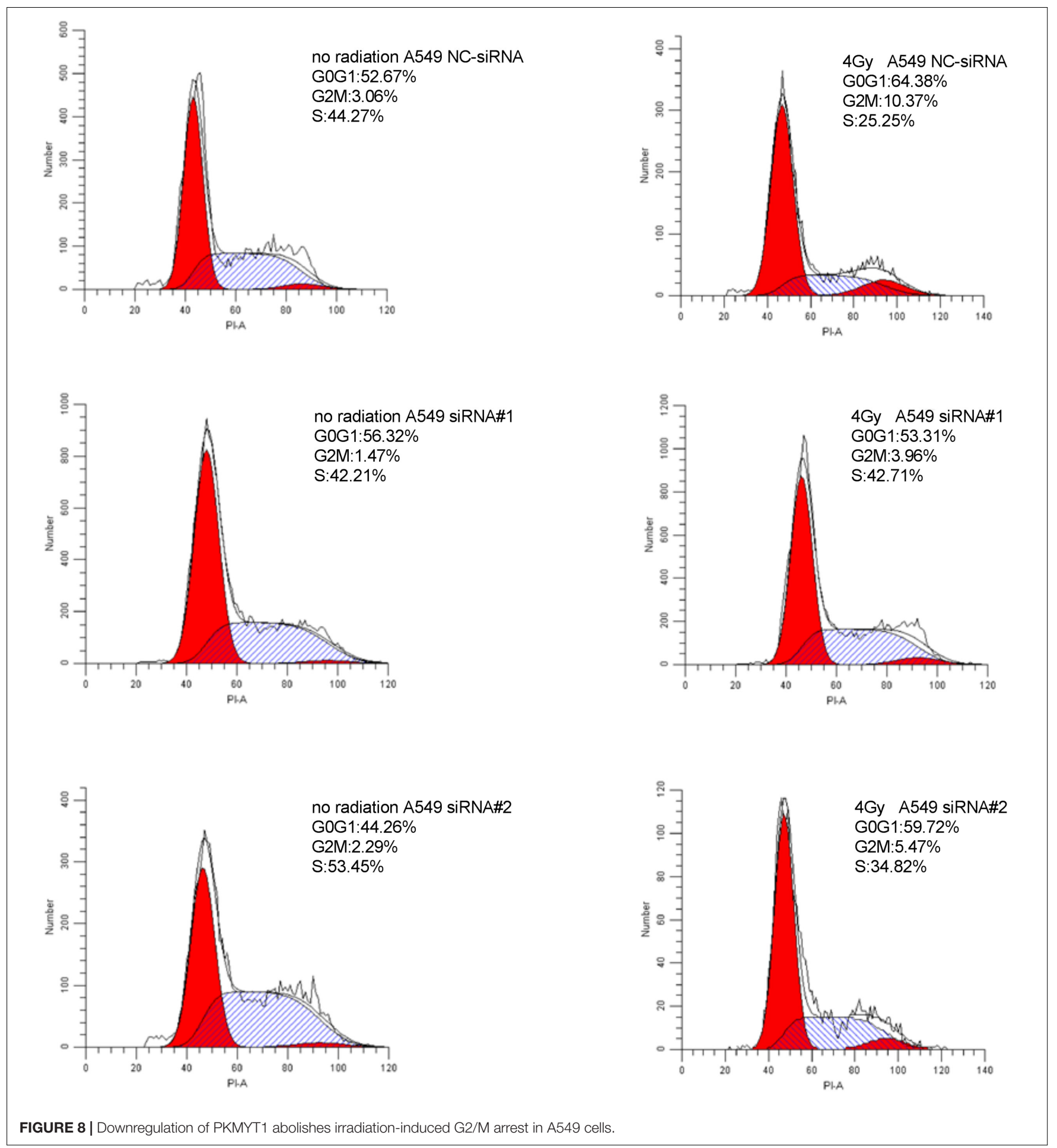

\section{DISCUSSION}

The identification of a target to improve the radiosensitivity of LUAD is significant. In this article, we obtained 14 core genes related to the prognosis of LUAD by analyzing GSE32863, GSE33532, and GSE43458. After an extensive literature review of these 14 genes, we found that PKMYT1 is a highly promising gene that may be closely related to tumor radiosensitivity. This is because PKMYT1 is currently considered to be the target of G2 checkpoint elimination and mitotic catastrophe (Tse et al., 2009; Schmidt et al., 2017) as it can prevent cells from transitioning from G2 to mitosis phase by affecting the activity of Cdk1 and the nuclear shuttle of the Cdk1-CycB complex (Fattaey and Booher, 1997; Wells et al., 1999; Passer et al., 2003). Additionally, the 

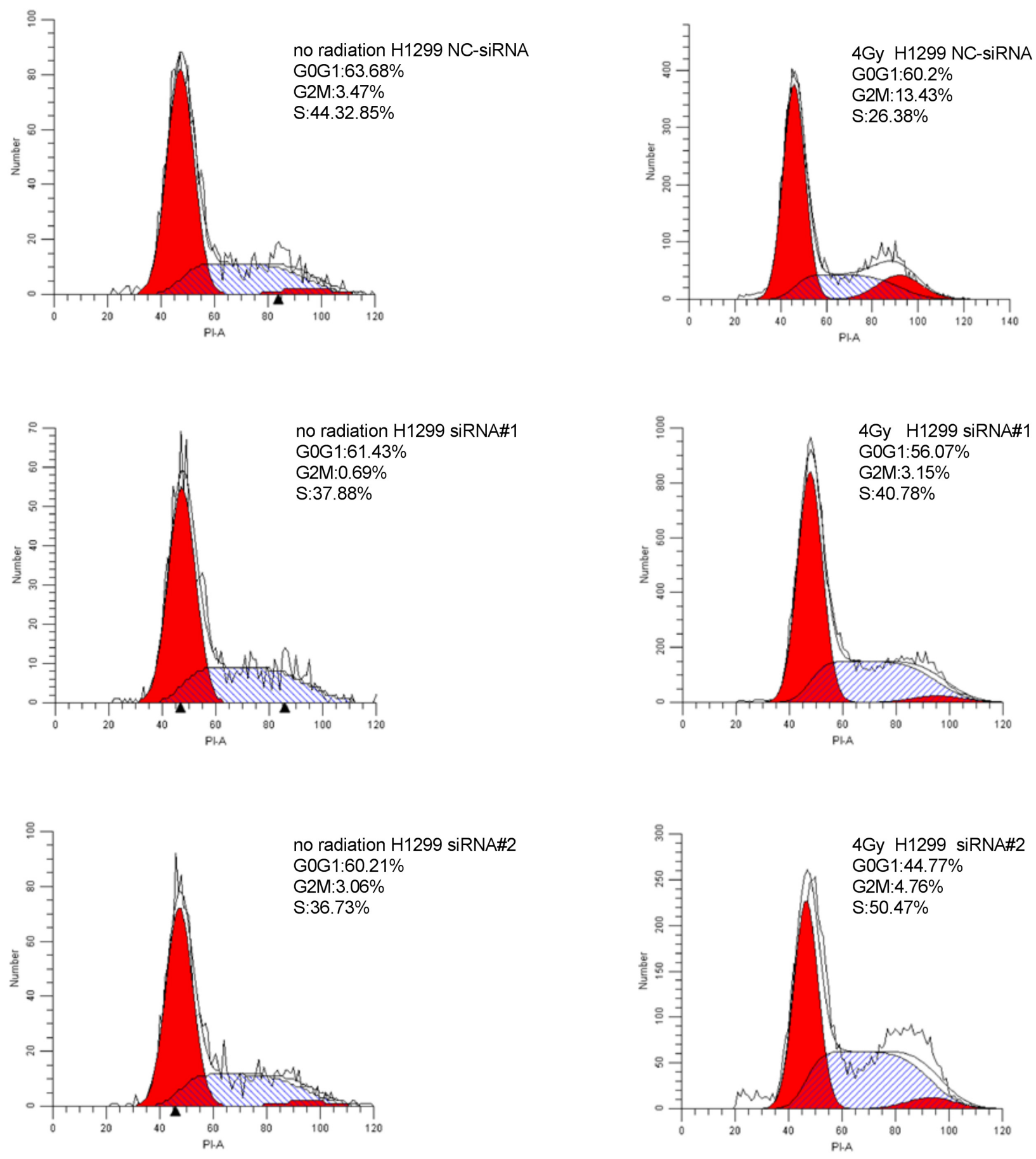

FIGURE 9 | Downregulation of PKMYT1 abolishes irradiation-induced G2/M arrest in H1299 cells.

abrogation of the G2 checkpoint effectively reduced radiationinduced cell cycle arrest and increased tumor radiosensitivity (Leijen et al., 2010; De Witt et al., 2011; Qin et al., 2014; Busch et al., 2017). However, the functional importance of PKMYT1 in tumor radiosensitivity has not yet been reported.

In this study we demonstrated that PKMYT1 is highly expressed in LUAD tissues contrasted with normal lung tissues.
We found that artificially silencing the expression of PKMYT1 can increase the sensitivity of LUAD cells to radiation. Cell cycle analysis found that downregulation of PKMYT1 alters cell cycle distribution and abrogates IR-induced G2/M phase arrest.

Most cancer types fail to establish the G1 checkpoint, and this is usually because of mutation or deletion of the p53 tumor suppressor. Hence, cells deficient in p53 are more vulnerable 
to inactivation of the G2 checkpoint (Passer et al., 2003). As a consequence, therapeutic ablation of G2 checkpoints may preferentially make p53-deficient tumor cells sensitive to DNA damaging treatments while retaining normal cells with intact p53 function (Dixon and Norbury, 2002; Meng et al., 2018). In addition, a very interesting point is that research has found that PKMYT1 is not necessary for the cell cycle of normal cells, but it has a rate-limiting function for the recovery of checkpoints after DNA damage (van Vugt et al., 2004; Chow and Poon, 2013). Inhibitors of components essential for the progression of cell cycle progression in normal cells may be limited by their greater toxicity to normal cells when subjected to anti-tumor therapy. Since PKMYT1 is relatively less important for normal cell cycle progression, PKMYT1 is a particularly attractive target for anticancer therapy.

\section{CONCLUSION}

In summary, we obtained 14 core genes (PKMYT1, TTK, CHEK1, CDC20, PTTG1, MCM2, CDC25C, MCM4, CCNB1, CDC45, MAD2L1, CCNB2, BUB1, and CCNA2) related to the poor prognosis of LUAD via bioinformatical analysis. We

\section{REFERENCES}

Bray, F., Ferlay, J., Soerjomataram, I., Siegel, R. L., Torre, L. A., and Jemal, A. (2018). Global cancer statistics 2018: GLOBOCAN estimates of incidence and mortality worldwide for 36 cancers in 185 countries. CA Cancer J. Clin. 68, 394-424. doi: 10.3322/caac.21492

Busch, C. J., Kroger, M. S., Jensen, J., Kriegs, M., Gatzemeier, F., Petersen, C., et al. (2017). G2-checkpoint targeting and radiosensitization of HPV/p16-positive HNSCC cells through the inhibition of Chk1 and Wee1. Radiother. Oncol. 122, 260-266. doi: 10.1016/j.radonc.2016.11.017

Chow, J. P., and Poon, R. Y. (2013). The CDK1 inhibitory kinase MYT1 in DNA damage checkpoint recovery. Oncogene 32, 4778-4788. doi: 10.1038/onc.2012. 504

De Witt, H. P., Mir, S. E., Noske, D., Van Noorden, C. J., and Wurdinger, T. (2011). WEE1 kinase targeting combined with DNA-damaging cancer therapy catalyzes mitotic catastrophe. Clin. Cancer Res. 17, 4200-4207. doi: 10.1158/1078-0432. CCR-10- 2537

Dixon, H., and Norbury, C. J. (2002). Therapeutic exploitation of checkpoint defects in cancer cells lacking p53 function. Cell Cycle 1, 362-368. doi: 10.4161/ cc.1.6.257

Fattaey, A., and Booher, R. N. (1997). Myt1: a Wee1-type kinase that phosphorylates Cdc2 on residue Thr14. Prog. Cell Cycle Res. 3, 233-240. doi: 10.1007/978-1-4615-5371-7_18

Le Pechoux, C. (2011). Role of postoperative radiotherapy in resected non-small cell lung cancer: a reassessment based on new data. Oncologist 16, 672-681. doi: 10.1634/theoncologist.2010-0150

Leijen, S., Beijnen, J. H., and Schellens, J. H. (2010). Abrogation of the G2 checkpoint by inhibition of Wee-1 kinase results in sensitization of p53deficient tumor cells to DNA-damaging agents. Curr. Clin. Pharmacol. 5, 186-191. doi: 10.2174/157488410791498824

Meng, X., Bi, J., Li, Y., Yang, S., Zhang, Y., Li, M., et al. (2018). AZD1775 increases sensitivity to olaparib and gemcitabine in cancer cells with p53 mutations. Cancers 10:149. doi: 10.3390/cancers 10050149

Passer, B. J., Nancy-Portebois, V., Amzallag, N., Prieur, S., Cans, C., Roborel, D. C. A., et al. (2003). The p53-inducible TSAP6 gene product regulates apoptosis and the cell cycle and interacts with Nix and the Mytl kinase. Proc. Natl. Acad. Sci. U.S.A. 100, 2284-2289. doi: 10.1073/pnas.0530298100 identified that PKMYT1 was significantly upregulated in LUAD tissues and firstly demonstrated that knockdown of PKMYT1 resulted lower survival rate when cells receiving radiation therapy. Our findings suggested that PKMYT1 is a promising target to improve the radiosensitivity of lung adenocarcinoma.

\section{DATA AVAILABILITY STATEMENT}

The datasets generated for this study can be found in the data is available at NCBI GEO under accession numbers GSE33532, GSE32863, and GSE43458.

\section{ETHICS STATEMEMT}

This study was approved by the Ethics Committee of The First Hospital of China Medical University.

\section{AUTHOR CONTRIBUTIONS}

HL analyzed the data and drafted the manuscript. JL, YY, QQ, and GL provided suggestions and approved the final manuscript.

Provencio, M., Sanchez, A., Garrido, P., and Valcarcel, F. (2010). New molecular targeted therapies integrated with radiation therapy in lung cancer. Clin. Lung Cancer 11, 91-97. doi: 10.3816/CLC.2010.n.012

Qin, Q., Cheng, H., Lu, J., Zhan, L., Zheng, J., Cai, J., et al. (2014). Smallmolecule survivin inhibitor YM155 enhances radiosensitization in esophageal squamous cell carcinoma by the abrogation of G2 checkpoint and suppression of homologous recombination repair. J. Hematol. Oncol. 7:62. doi: 10.1186/ s13045-014-0062-8

Schmidt, M., Rohe, A., Platzer, C., Najjar, A., Erdmann, F., and Sippl, W. (2017). Regulation of G2/M transition by inhibition of WEE1 and PKMYT1 kinases. Molecules 22:2045. doi: 10.3390/molecules22122045

Siegel, R. L., Miller, K. D., and Jemal, A. (2018). Cancer statistics, 2018. CA Cancer J. Clin. 68, 7-30. doi: 10.3322/caac. 21442

Tse, A. N., Sheikh, T. N., Alan, H., Chou, T. C., and Schwartz, G. K. (2009). 90-kDa heat shock protein inhibition abrogates the topoisomerase I poison-induced G2/M checkpoint in p53-null tumor cells by depleting Chk1 and Wee1. Mol. Pharmacol. 75, 124-133. doi: 10.1124/mol.108.05 0807

van Vugt, M. A., Bras, A., and Medema, R. H. (2004). Polo-like kinase-1 controls recovery from a G2 DNA damage-induced arrest in mammalian cells. Mol. Cell. 15, 799-811. doi: 10.1016/j.molcel.2004.07.015

Wells, N. J., Watanabe, N., Tokusumi, T., Jiang, W., Verdecia, M. A., and Hunter, T. (1999). The C-terminal domain of the Cdc2 inhibitory kinase Myt1 interacts with $\mathrm{Cdc} 2$ complexes and is required for inhibition of $\mathrm{G}(2) / \mathrm{M}$ progression. J. Cell Sci. 112(Pt 19), 3361-3371.

Conflict of Interest: The authors declare that the research was conducted in the absence of any commercial or financial relationships that could be construed as a potential conflict of interest.

Copyright (c) 2020 Long, Liu, Yu, Qiao and Li. This is an open-access article distributed under the terms of the Creative Commons Attribution License (CC BY). The use, distribution or reproduction in other forums is permitted, provided the original author(s) and the copyright owner(s) are credited and that the original publication in this journal is cited, in accordance with accepted academic practice. No use, distribution or reproduction is permitted which does not comply with these terms. 